\title{
A Case Report on Pneumonic Pasteurellosis in Goats at Organized Farm of Madhya Pradesh
}

\author{
G.P. Jatav ${ }^{1}$, Nidhi Shrivastava ${ }^{1}$, A.K. Jayraw ${ }^{2}$, M. Shakya ${ }^{2}$, S. Shukla ${ }^{1}$ and S.D. Audarya ${ }^{3}$ \\ Department of Veterinary Pathology \\ College of Veterinary Science and Animal Husbandry, Nanaji Deshmukh Veterinary Science University, \\ Mhow- 453 446, Indore, Madhya Pradesh, India \\ ${ }^{1}$ Department of Veterinary Pathology \\ ${ }^{2}$ Department of Veterinary Parasitology \\ ${ }^{3}$ Department of Veterinary Microbiology
}

\begin{abstract}
An outbreak of pasteurellosis in goats was investigated at an organized goat farm from Nimar region of Madhya Pradesh. The farm visited to investigate the cause of deaths in goats which exhibited the signs like dullness, lethargy, high fever, dyspnea and profuse salivation and death. Post-mortem examination revealed lesions like haemorrhagic tracheitis, lung emphysema, pin point haemorrhages on myocardial surface, congestion in abomasum, small intestine with pin point haemorrhages, oedematous mesenteric lymph nodes and friable liver. Blood smear examination of morbid goats revealed presence of Pasteurella multocida. Hence, therapeutic management with oxytetracycline resulted in recovery of infected animals.
\end{abstract}

Keywords:- Goats; Madhya Pradesh; Oxytetracycline; Pasteurella multocida; Pneumonic pasteurellosis.

\section{INTRODUCTION}

In India, haemorrhagic septicaemia (HS) caused by Pasteurella multocida is the most important bacterial contagious disease of ruminants. It causes acute infection in large and small ruminants which is responsible for heavy mortality. The $P$. multocida causes HS in cattle and buffaloes while pneumonic pasteurellosis in sheep and goats as they are commonly found in tonsils and nasopharynx of healthy sheep and goats. Although it is a very important disease of buffaloes, it has been reported in goats with an outbreak rate of $62 \%$ (2007-2010). This disease is common in farms with poor managemental practices and observed as a secondary bacterial infection. Routinely, it is diagnosed based on clinical symptoms and post-mortem lesions, and it is confirmed by isolation and identification of bipolar organisms. Antibiotics like penicillin, ampicillin, tetracycline, oxytetracycline, tylosin, florfenicol and ceftiofur have been effectively used for treating pneumonic pasteurellosis in goats (Maria, 2007). The causative agent of HS has got zoonotic significance (Aski \& Tabatabaei, 2016). The present communication reports the pneumonic pasteurellosis in organised goat farm of Madhya Pradesh.

\section{MATERIALS AND METHODS}

In the present study, the outbreak of pasteurellosis was investigated in an organized goat farm situated in Nimar region of Madhya Pradesh. The animals were reared in isolated fences with 30-40 goats per fence. The outbreak was recorded in mid winter (last - December) 2014 in one band (a group of goats) comprising Jamunapari and Barbari breeds of goats having different age group and these goats were vaccinated against haemorrhagic septicaemia. At this goat farm, more than $50 \%$ morbidity with increased death rate among the morbid goats with $10-15 \%$ mortality was recorded within 24-48 hours from the initial occurrence of the illness. The present investigation recorded the major clinical signs like dullness, lethargy, high fever (up to $\left.106^{\circ} \mathrm{F}\right)$, dyspnea and profuse salivation. After clinical examination of the affected animals, aseptically blood samples were collected from 5 suspected goats for blood smear examination. Post-mortem examination of dead animals was conducted and gross pathological lesions of different organs were documented (Fig. 1).

\section{RESULTS AND DISCUSSION}

Blood smears were prepared from the collected blood of infected goats which were fixed by heat and stained with Leishmann's stain. Blood smear examination revealed presence of Gram negative, bipolar organisms indistinguishable between Pasteurella multocida and Mannhaemia haemolytica reported earlier from the blood samples. The post-mortem lesions like congested trachea, emphysematous lungs, petechial haemorrhages in the myocardium, congested abomasum, focal petechial haemorrhage in small intestine, swollen or congested mesenteric lymph nodes and congested and fragile liver were recorded (Fig. 1). Similarly, Jesse Abdullah et al. (2014) also observed pneumonic pasterellosis in goat from Malaysia.

The remaining morbid animals were treated with oxytetracycline, the drug of choice for pasteurellosis, intravenously with normal saline and intramuscularly with long acting oxytetracycline. The treated goats recovered within a week and no further mortality was recorded. Leishman stained blood smears revealed presence of bipolarity, which is a specific character of Pasteurella spp. Morphological and staining characteristics along with the 
therapeutic response to the treatment indicated involvement of the organism Pasteurella multocida or Mannhaemia haemolytica.

\section{CONCLUSION}

The present study revealed pneumonic pasteurellosis caused by Pasteurella multocida as a cause of death at organized goat farm of Madhya Pradesh. The infection was successfully treated using oxytetracycline with recovery of infected animals.

\section{REFERENCES}

[1]. Aski H.S. and Tabatabaei, M. (2016). Occurrence of virulence associated genes in Pasteurella multocida isolates obtained from different hosts. Microbial Pathogenesis pii: S0882-4010(15)30093-0.

[2]. Jesse Abdullah, F.F., Tijjani, A., Adamu, L., Teik Chung, E.L., Abba, Y., Mohammed, K., Saharee, A.A., Haron, A.W., Sadiq, M.A., Mohd, A.M.L. (2014). Pneumonic pasteurellosis in a goat. Iranian Journal of Veterinary Medicine, 8(4):293-296.

[3]. Maria, L. (2007). Bacterial pneumonia in goats. In: Alabama Cooperative Extension System online publication. www.aces.edu/urban.

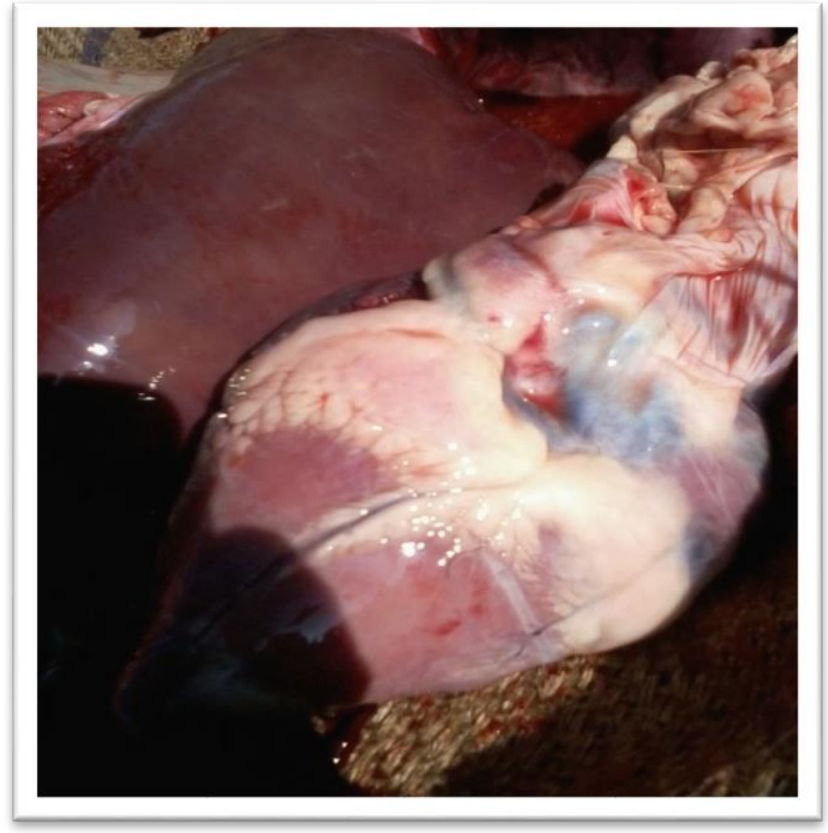

(a)

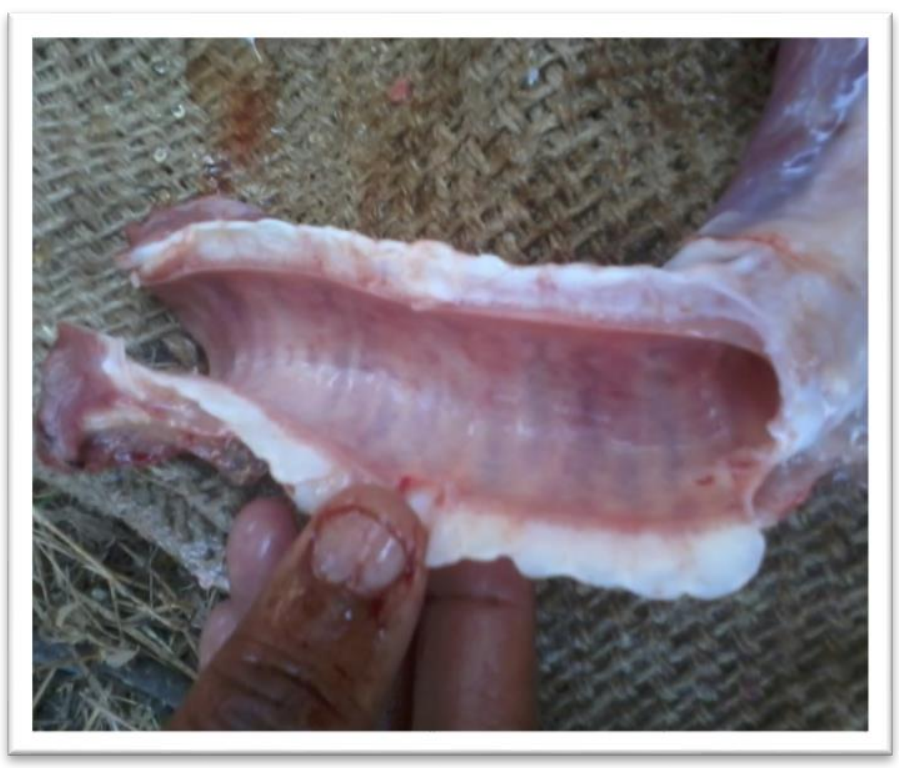

(b)

Fig 1:- Petechial haemorrhages on the heart and haemorrhagic tracheitis 